\title{
Smart HIV Testing System
}

\author{
Ali El Kateeb, ${ }^{1}$ Peter LaW, ${ }^{1}$ and KIng CHAN ${ }^{2}$ \\ ${ }^{1}$ Electrical and Computer Engineering Department, University of Michigan, Dearborn, MI; \\ ${ }^{2}$ Medmira Inc., Halifax, Nova Scotia, Canada \\ (Received 9 June 2004; accepted 4 January 2005)
}

\begin{abstract}
The quick HIV testing method called "MiraWell Rapid HIV Test" uses a specialized testing kit to determine whether an individual's blood is contaminated with the HIV virus or not. When a drop of blood is placed on the center of the testing kit, a simple pattern will appear in the middle of the kit to indicate the test status, i.e., positive or negative. This HIV test should be done in a small clinic or in a lab and the test must be conducted by a trained technician. A smart HIV testing system was developed through this research to eliminate the human error that is associated with the use of the quick HIV testing kits. Also, the smart HIV system will improve the testing productivity in comparison to those achieved by the trained technicians.

In this research, we have developed a cost-effective system that analyzes the image produced by the HIV kits. We have used a System-On-Chip (SOC) design approach based on the Field Programmable Gate Array (FPGA) technology and the Xilinx Virtex SOC chip in building the system's prototype. The system used a CMOS digital camera to capture the image and an FPGA chip to process the captured image and send the testing results to the display unit. The system can be used in small clinics and pharmacies and eliminates the need for trained technicians. The system has been tested successfully and $98 \%$ of the tests were correct.
\end{abstract}

Keywords-Quick HIV kits, Field programmable gate array (FPGA), HIV screening, System-on-chip (SOC).

\section{INTRODUCTION}

The HIV blood test is usually done in hospitals and could take some time before the patient knows the result. Other quick HIV test is performed through the use of special kits that produce a certain pattern indicating the status of the blood condition. ${ }^{1,3}$ Such quick tests should be performed by a trained technician and may be conducted in a small clinic or lab. The test results can be obtained in a minute.

As an example, one of these quick HIV kits has two bars to indicate where the test is conducted correctly and also indicate whether the test's result was positive or negative. ${ }^{1}$

Address correspondence to Ali El Kateeb, Electrical and Computer Engineering Department, The University of Michigan, 4901 Evergreen Road, Dearborn, MI 48128-1491. Electronic mail: elkateeb@umd.umich.edu
The kit has a control bar that appears when the testing procedure is conducted correctly. The other bar is called the testing bar, which indicates the testing result (Fig. 1). When a drop of blood is placed on the center of the testing kit, a testing bar pattern will appear in the middle of the testing kit. The testing bar appears due to the reaction between the chemicals that exist in the kit and the blood. The appearance of the testing bar will indicate that the result of the test is positive and the blood is contaminated with the HIV virus. Otherwise, the test is negative and the testing bar will not appear on the kit.

The darkness appearance of the testing bar is usually varied between very light and very dark. Also, the bar may not appear fully on the testing kit; sometimes only a fraction of the testing bar will appear. Such variations in the appearance of the testing bar have made the determination of the testing result hard in some cases. For example, when the intensity of the bar is so low it could make technicians interpret the results differently. It is important to have very accurate testing because the false positive result will affect the patient psychologically. It can also cause serious harm; a patient who has received a false negative result will continue to infect other people not knowing that he/she carries the HIV virus.

The objective of this research is to develop a smart system that is capable of eliminating human errors and improving testing accuracy. The system can analyze the image generated by the testing kit and provide the accurate results in seconds. Moreover, producing a self-operated smart system that analyzes the testing kit will make it possible to perform such testing at the remote places where the trained technicians may not be available. The availability of an accurate and cost effective system for HIV testing may allow using such systems by the patients themselves in pharmacies or small clinics without the technicians support. Clearly, that will improve the HIV screening process.

\section{DESIGN CONSIDERATION}

In order to perform the processing of the image that is produced by the HIV testing kits, it is required to capture the 


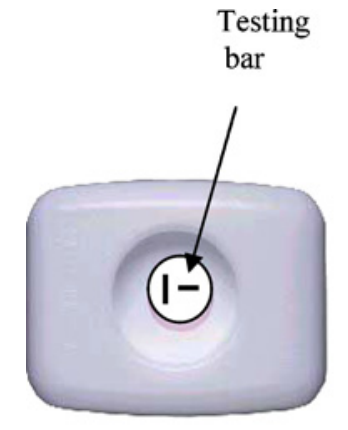

Positive

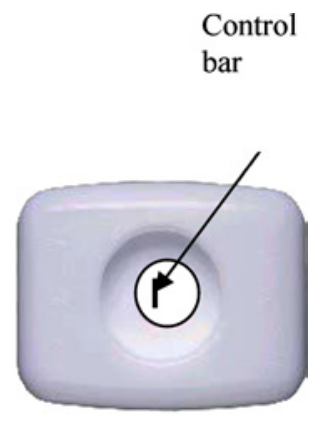

Negative
FIGURE 1. HIV test kit.

image of the testing result first. We decided not to use offthe-shelve frame grabber unit but to design and implement such unit that can be integrated easily on a single chip with the rest of the hardware that is required by the HIV smart system. A CMOS digital camera sensor has been used to capture the image from the HIV kit. Such sensor is inexpensive and provides an accurate digital image that can be easily stored by the sensor interface unit of the smart system for further processing. The Black and White sensor M4088 is used in this work where it produced an image of 385 by 288 pixels size. Each pixel is 8-bit. Every captured pixel is stored in the smart system's frame buffer, which is a static memory unit of a size enough to hold a full captured image of the testing result.

To have a cost effective design, a System-On-Chip (SOC) design approach is used in this project. This design approach could integrate the processing core, CMOS sensor interface, display interface for the test results, and frame buffer interface on a single chip. The SOC that is based on reconfigurable technology, specifically the Field Programmable Gate Array (FPGA) technology, was used in this research. The reconfigurable technology will provide the design with the required flexibility. As these HIV quick testing kits are new, it is expected that the technology and the design of such kits could be changed frequently. For instance, one of these available quick HIV testing kits has been enhanced after first release of the HIV kit product. In the first release of the product, a kit with a dot image was used (Fig. 2). In the second version of the product, an enhancement was achieved by adding a control bar to indicate whether the test conducted correctly or not (Fig. 3). The testing dot is changed into two bars. The images shown in Figs. 2 and 3 are captured by the CMOS sensor that is used by the smart system.

The design flexibility achieved by using reconfigurable technology will allow adjusting the system to enhance new modifications that could occur to the testing kit without changing the system. As the new version of the kit is released, the reconfiguration chip could be modified to support the HIV kit's new features. ${ }^{2,4}$ The sys-

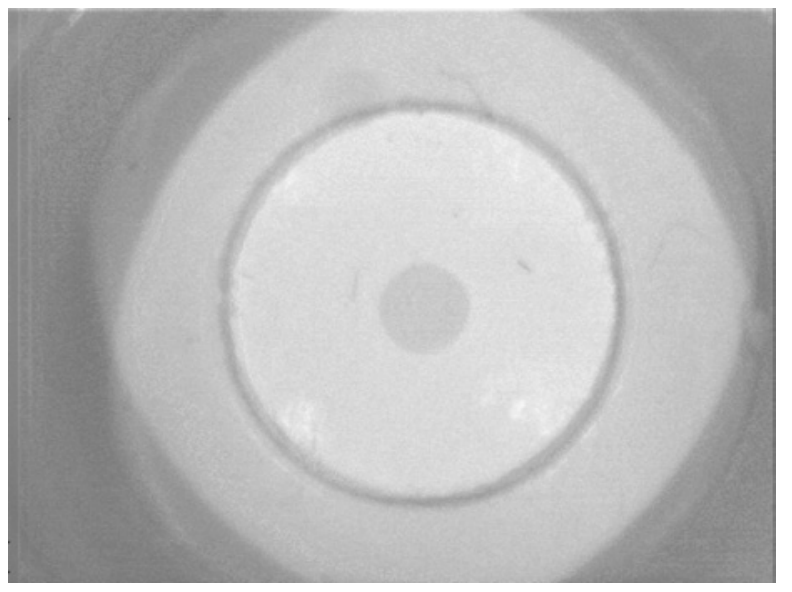

FIGURE 2. First version of quick HIV testing kit.

tem based on embedded microcontroller could also adapt the kit's changes by reprogramming the microcontroller. However, the microcontroller-based system will need more than one chip in implementing the whole system. In addition, the change that occurs to the subsystem interface will require redesigning the interface and therefore reimplementing the system will be costly and a time consuming process. Moreover, the system implementation using Application Specific Integrated Circuit (ASIC) will be costly since a new ASIC has to be designed whenever a new version of the HIV kit becomes available.

The use of the FPGA-based reconfigurable chip will reduce the system development cost since the system processing core and other required hardware will be implemented on one chip. We have used a Xilinx Virtex (XCV50-PQ240) SOC chip in the system implementation. ${ }^{5}$ This chip does not have enough buffers to save the captured image and hence an external memory has been used to store the image on an external SRAM buffer. The FPGA provides the processing power and the hardware required for this system.

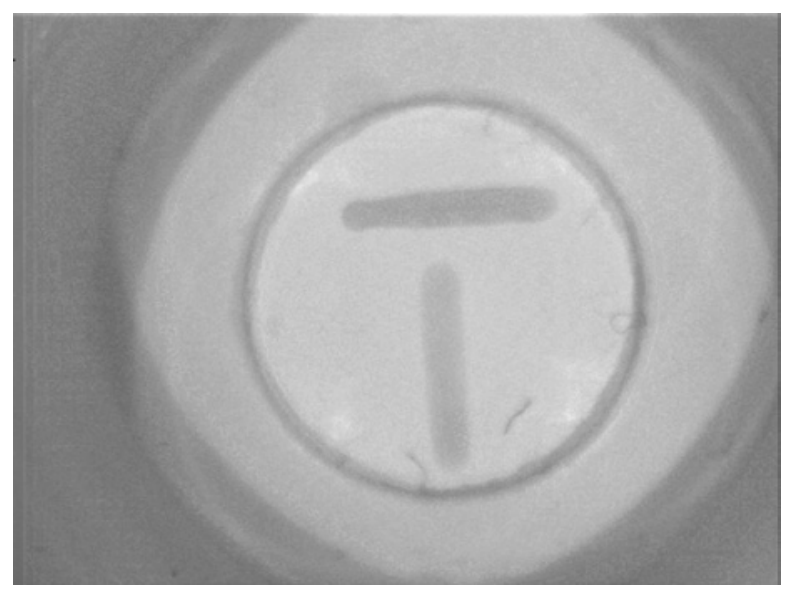

FIGURE 3. Second version of the quick HIV testing kit. 


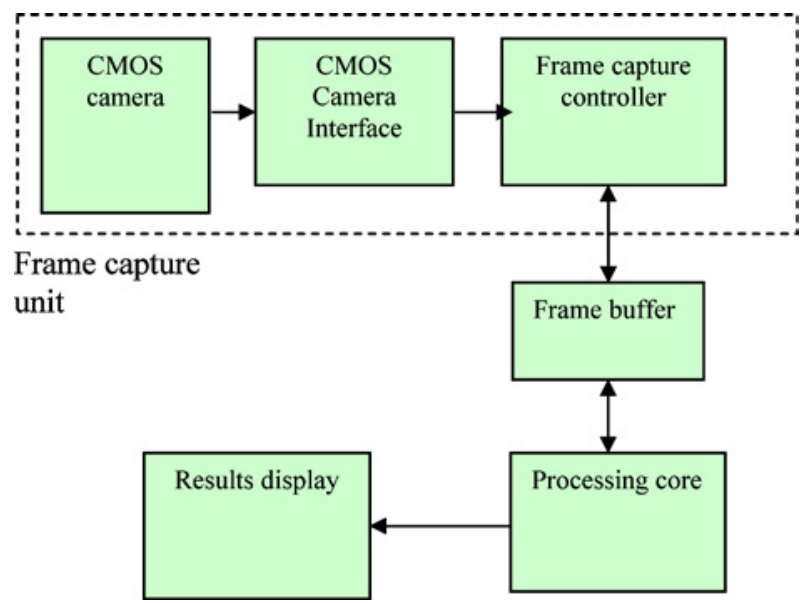

FIGURE 4. Smart HIV system structure.

\section{HIV SMART SYSTEM DESIGN}

The smart HIV system design includes the following main units (Fig. 4):

1. Frame capture, which includes CMOS camera, CMOS camera interface and frame capture controller.

2. Processing core.

3. Results display.

4. Frame buffer.

The CMOS camera, the frame buffer, and the display unit are all off-the-shelve components. All other units are implemented with the FPGA chip. The type of the camera is a CMOS sensor M4088. The camera interface has buffers to store every captured pixel and control all sensor signals. The frame capture part is to allow the capturing of the whole image from the testing kit and to save those captured pixels in the frame buffer. As the sensor provides 50 frames per second, only one of these frames is needed by the HIV system. Therefore, frame controller is designed to read one image and store it in a frame buffer. The frame buffer size is 110192 bytes. When a row of pixels is completely captured and stored in the frame buffer, the frame controller will capture the next row and store it in the frame buffer after the first row. This process will continue until the last row of the frame is stored, i.e., row number 288 . When one full frame is stored, the image capture unit will trigger the processing core to start analyzing the image.

The CMOS sensor has a $1 / 3^{\prime \prime}$ lens focused on the center of the kit, where the test and control bars are located. The CMOS sensor is placed in a specially designed cartridge that blocks the outside light from getting into the cartridge. By doing so, a uniform light is directed to the HIV kit providing a better result for capturing the image of the kit (Fig. 5). The CMOS sensor lens has been supported with the illumination system. This system has four Light Emitting Diodes (LED) that are located around the lens of the CMOS camera to distribute the light uniformly over the image of the HIV kit (Fig. 6).

\section{HIV IMAGE PROCESSING}

The image produced by the HIV kit is stored in the frame buffer. That image is scanned to detect whether the test is positive or negative. Different possible methods

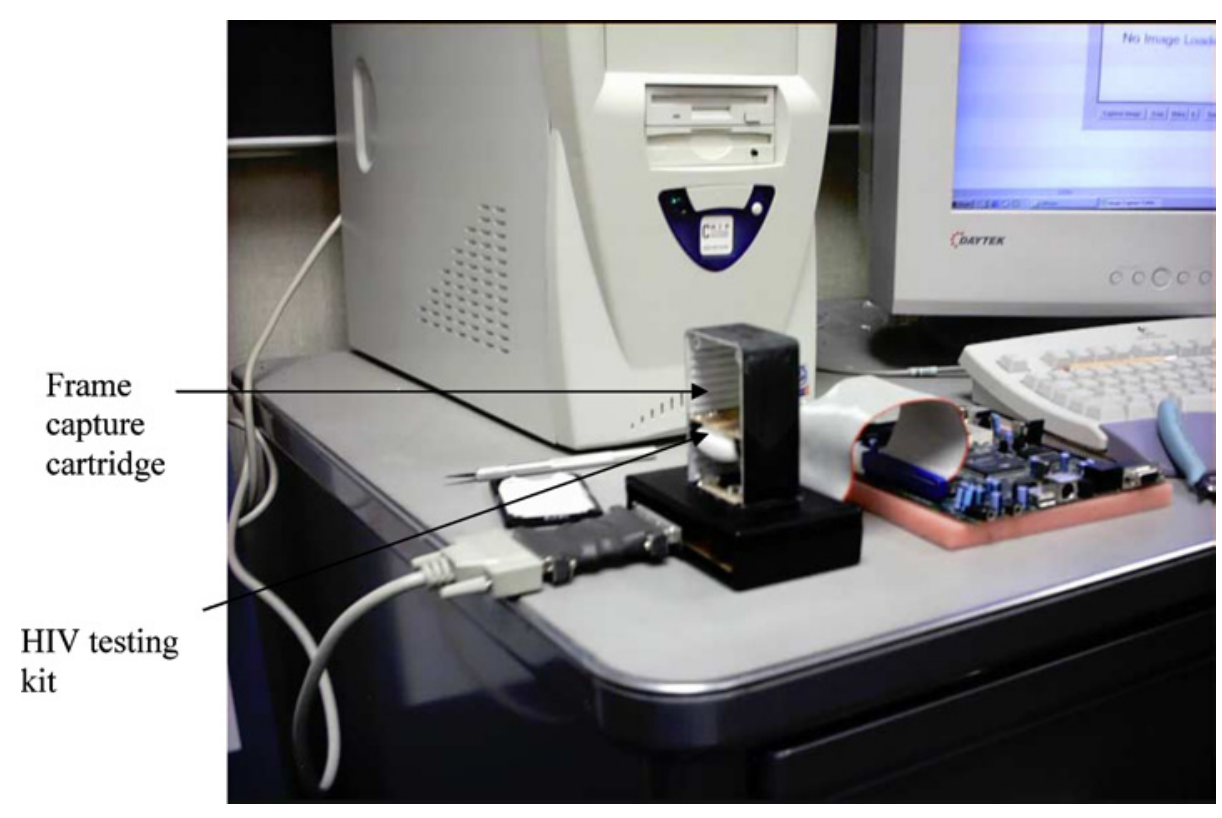

FIGURE 5. Smart HIV testing system prototype. 


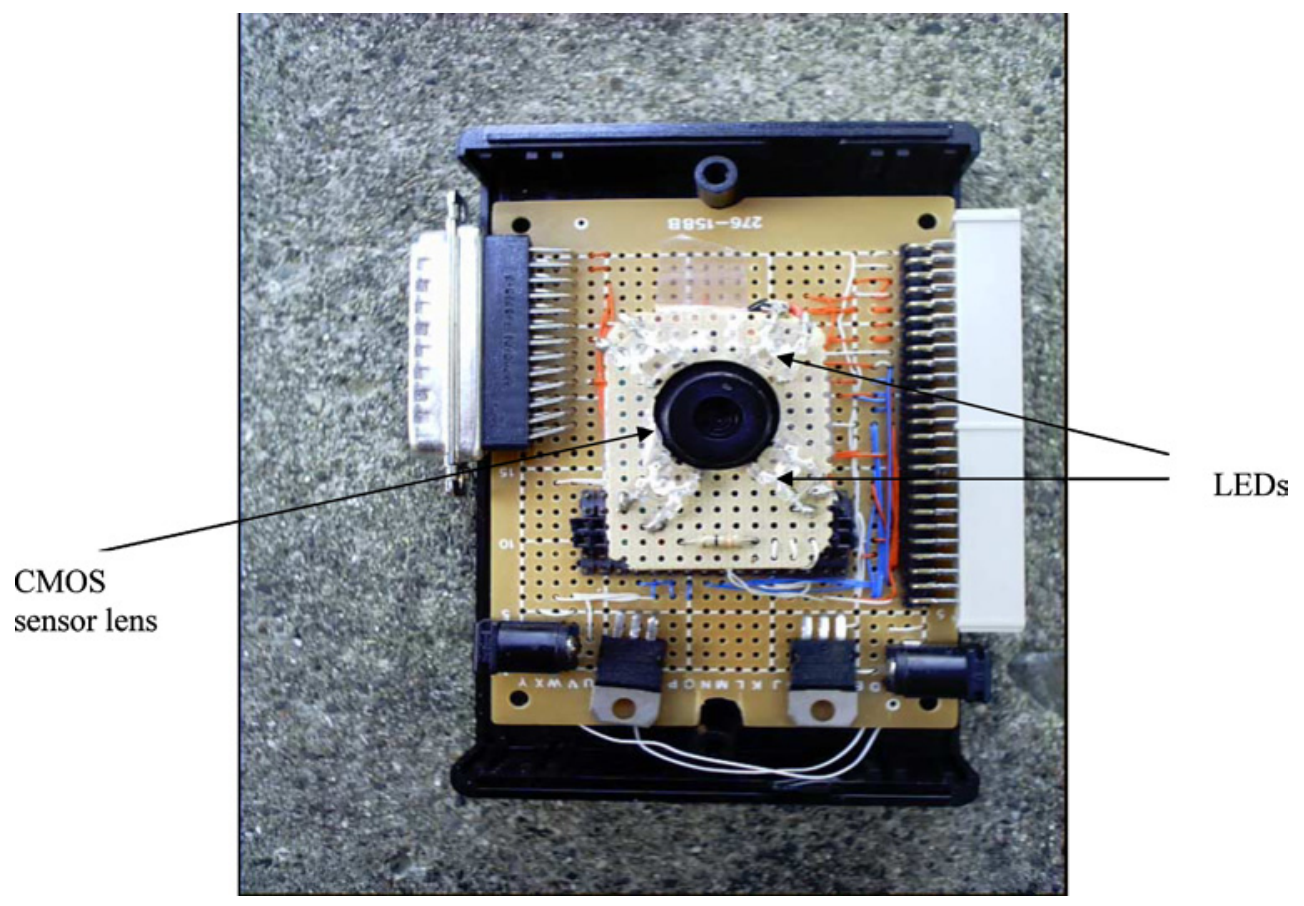

FIGURE 6. LEDs around the CMOS sensor lens.

can be used to perform the image processing. However, we selected a simple method that checks the existence of the horizontal (control) bar as well as the intensity of the color and the existence of the vertical (testing) bar. The CMOS sensor lens has been focused, during the system integration, on the HIV kit's area that contains the control and testing bars. Since the HIV kit is located in the same position in the cartridge (Fig. 5), the control and testing bars will be located at the same place in the captured image. Clearly, that will be applied for all captured kits' images.
The processing core will then determine the average pixel value for the image background (Fig. 7). The average pixel value has been measured by measuring the average value for all the pixels located in small area, with a size of $64 \times 64$ pixels, on the image background. This area is selected to be far from the testing and control bars. The average pixel value is used to determine the intensity of the pixels located in control and testing bars. The processing core scans the area where the control and testing bars are located. All pixels in that area with a value greater than the average background pixels are considered as valid pixels

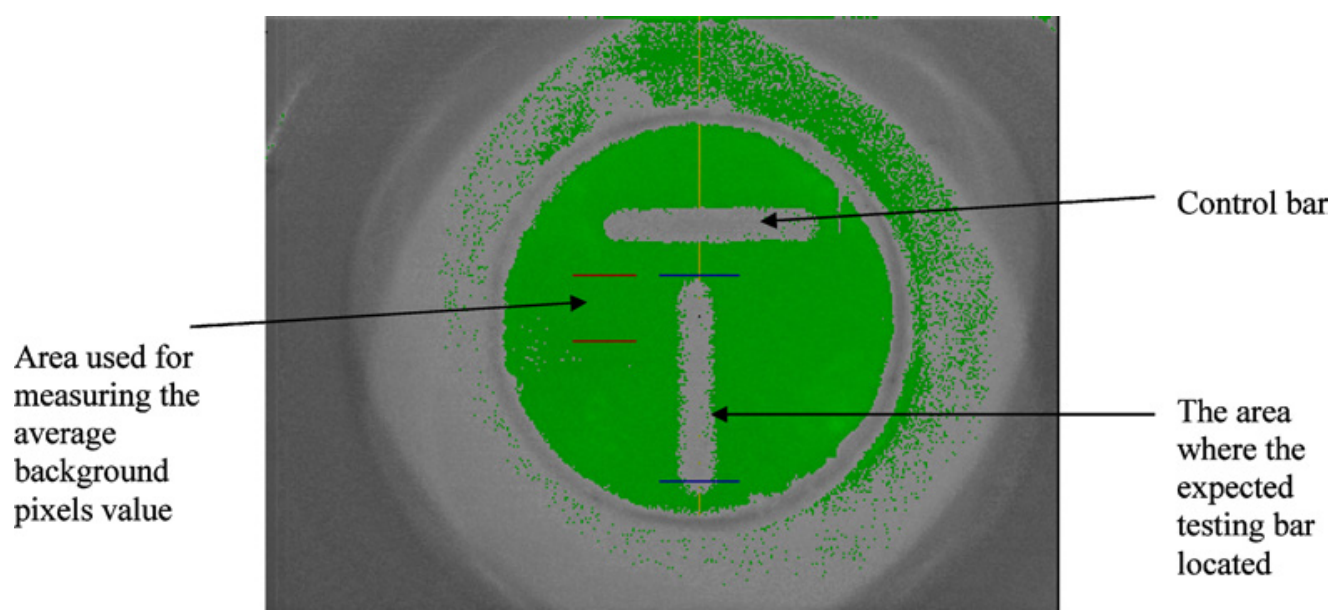

FIGURE 7. Location of the area for measuring the average background pixels value and the area where the testing bar located. 


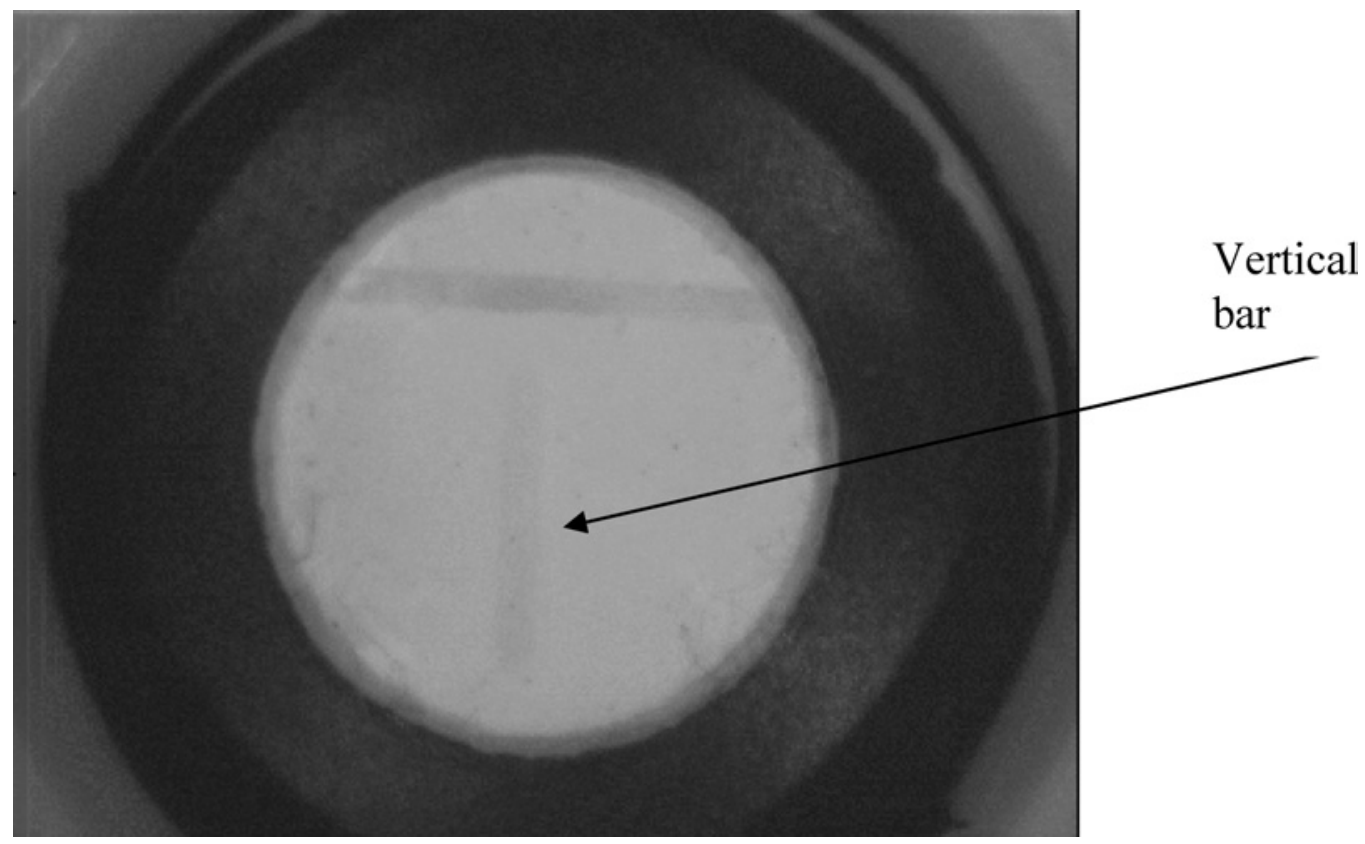

FIGURE 8. A positive test with incomplete bar.

of the control and testing bars. The detection of a hundred pixels in the area where the control bar located indicates the existence of the control bar. That is, the test is conducted correctly.

Figure 7 shows the detected bars after the image is captured where we changed the color of the background to green and the bars' color is displayed with gray. Clearly, Fig. 7 indicates that the method used in this work would detect the bars accurately.

In some testing cases, the testing bar is not appeared clearly due to the fact that a chemical reaction with the blood produces an incomplete and light-colored bar (Fig. 8). The method that is used in the bar analysis has took such cases into account. The processing core indicates a positive case if 20 horizontal lines of the testing bar have a higher pixel value than the average background pixel value. Detecting less than 20 lines will result in displaying a message requesting the technician to repeat the test. The case where more than 20 lines of testing bar are detected will indicate the existing of a positive testing case. Otherwise the test result is negative. This threshold of the number of appeared lines have been selected to avoid providing a positive result based on the appearance of a shadow on the image captured because of the light reflection on the kit's image surface. The pixels in the shadow area have higher pixel value than the average pixel value, and hence provide a wrong testing result.

We have used seven segment displays to indicate the negative and the positive results to the technicians. Other messages are also supported by the system such as informing the technician to repeat the test or consulting a spe- cialist where the results are hard to be interpreted by the system's processing core. Such a case could happen when two consecutive tests produces less than 20 lines of the testing bar.

The accuracy of the system has been tested by using HIV kits that were collected from real tests conducted on many patients. We have found that the smart system provide an accurate results for $90 \%$ of the tests when such tests are conducted for the first time. As the testing repeated for those $10 \%$ of the failed tests, the system is able to detect additional $6 \%$ correctly. Only $4 \%$ of the whole tests had to be conducted by technician to provide accurate results for these remaining HIV kits. The smart system completes the test in less than a second.

The system accuracy and the simplicity of using the system would encourage utilizing the system in small clinics and pharmacies and without a trained technician support. That will improve the screening process since the availability of the system will be very high, which encourage patient to conduct such test frequently and whenever there is a need for it.

\section{CONCLUSION}

In this work, we have successfully designed and implemented an HIV testing system that analyzes the images produced by the quick HIV testing kits. The system provides the users with the messages about test results such as positive, negative, repeat test, consult physician and error messages. The system use reconfigurable technology that can be easily reprogrammed to update the system to keep 
up with new type of HIV kits, new processing approach, new sensors cameras, etc. Although the smart HIV system has many units shown in Fig. 4, only three units are designed with the FPGA chip, which are CMOS camera interface, frame capture controller, and processing core. The system has been tested and the result matches those results that were performed by the expert in the HIV testing field.

\section{REFERENCES}

${ }^{1}$ MedMira Laboratory web page: www.medmira.com.

${ }^{2}$ Nekoogar, F., and F. Nekoogar. From ASICs to SOCs. Englewood Cliffs, NJ: Prentice Hall, 2003.

${ }^{3}$ OraSure Technologies Inc. web page: www.orasure.com.

${ }^{4}$ Rajsuman, R. System-on-Chip Design and Test. Santa Clara, CA: Artech House Publisher, 2000.

${ }^{5}$ Xilinx web page: www.xilinx.com. 